\title{
Article
}

\section{Variability in Laboratory vs. Field Testing of Peak Power, Torque, and Time of Peak Power Production Among Elite Bicycle Motocross Cyclists}

P. Rylands, Lee, Roberts, Simon J. and Hurst, Howard Thomas

Available at http://clok.uclan.ac.uk/22170/

P. Rylands, Lee, Roberts, Simon J. and Hurst, Howard Thomas ORCID: 00000001-7889-8592 (2015) Variability in Laboratory vs. Field Testing of Peak Power, Torque, and Time of Peak Power Production Among Elite Bicycle Motocross Cyclists. Journal of Strength and Conditioning Research, 29 (9). pp. 2635-2640. ISSN 1064-8011

It is advisable to refer to the publisher's version if you intend to cite from the work. http://dx.doi.org/10.1519/JSC.0000000000000884

For more information about UCLan's research in this area go to http://www.uclan.ac.uk/researchgroups/ and search for <name of research Group>.

For information about Research generally at UCLan please go to http://www.uclan.ac.uk/research/

All outputs in CLoK are protected by Intellectual Property Rights law, including Copyright law. Copyright, IPR and Moral Rights for the works on this site are retained by the individual authors and/or other copyright owners. Terms and conditions for use of this material are defined in the policies page. 


\section{Title: Variability in laboratory versus field testing of peak power, torque and time of peak power production amongst elite BMX cyclists}

\section{Lead / Corresponding Author}

Lee Rylands

College of Life and Natural Sciences

\section{Contact Details}

Postal Address: LS/206 Lismore Villas, Devonshire Campus, Devonshire

Road, Buxton, Derbyshire, SK17 6RY

Telephone: $\quad 01332594437$

E-mail address: L.rylands@derby.ac.uk

\section{Co-authors}

Dr Simon Roberts

Faculty of Education, Community and Leisure, Liverpool John Moores

University

\section{Contact Details}

Postal Address: $\quad$ IM Marsh, Barkhill Road, Mossley Hill, Aigburth, L17 6BD

Telephone: 01512315426

E-mail address:

\section{S.Roberts2@ljmu.ac.uk}

\section{Co-authors}

Dr Howard Hurst

Division of Sport, Exercise and Nutritional Science

\section{Contact Details}

Postal Address: $\quad$ University of Central Lancashire, Darwin Building, DB223,

Preston, Lancashire, PR1 2HE

Telephone: +44 (0) 1772893911

E-mail address: HTHurst@uclan.ac.uk 


\section{ABSTRACT}

The aim of this study was to ascertain the variation in elite male BMX cyclists' peak power, torque and time of power production during laboratory and fieldbased testing. Eight male elite BMX riders volunteered for the study and each rider completed 3 maximal sprints using both an SRM ergometer in the laboratory, and a portable SRM power meter on an Olympic standard indoor BMX track. The results revealed a significantly higher peak power $(p=<0.001$, $34 \pm 9 \%)$ and reduced time of power production $(p=<0.001,105 \pm 24 \%)$ in the field tests when compared to laboratory derived values. Torque was also reported to be lower in the laboratory tests, but not to an accepted level of significance $(p=0.182,6 \pm 8 \%)$.

These results suggest that field based testing may be a more effective and accurate measure of a BMX rider's peak power, torque and time of power production.

Keywords: Bicycle motocross, peak power, time of power production, torque. 


\section{INTRODUCTION}

Confidence in the reliability and validity of measurement data gained from the laboratory testing of athletes is crucial if coaches are to develop sustained improvements in athletic performance. In sports such as elite level cycling it is well known that the difference between first and second place can be marginal (15) and therefore any objectively collected laboratory data needs to accurately reflect the demands of the race environment.

In cycle sports, including Bicycle motocross (BMX), the availability of reliable field-based testing equipment such as Schoberer Rad Messtechnik (SRM) power meters (25), timing gates (24) and mobile metabolic system (8) has led some researchers to question the exclusive use of laboratory based testing and have argued that field testing may be a more relevant method of analysis (11). For the past two decades cycling studies have demonstrated that a strong relationship exists between performance data obtained both in the laboratory and in the field $(13,2,19)$. However, despite the ecological validity of laboratory based testing acknowledged within cycling as a whole, there still remains some dispute between individual cycling disciplines $(20,22,9)$.

For instance Jobson et al. (17) investigated the ecological validity of laboratory testing in 23 competitive road cyclists. Velocity was analysed in two 25 mile time trials, one completed on a road course the other on a king cycle ergometer. The results revealed a $4 \%$ lower velocity recording on the road when compared to the laboratory test, which the authors found to be statistically significant. Interestingly, this $4 \%$ difference only occurred within 
riders that had a greater frontal surface area and it was concluded that the environmental factor of drag coefficients had an influence on the final test data.

Moreover, research conducted to examine the influence of drag between sprints performed in a laboratory and those of riders in an indoor gymnasium reported a $4 \%$ decrease in peak power between the two testing environments (3). The research also compared the differences in seated and standing sprints of both environments. Bertucci et al. (3) established a $32 \%$ higher force during standing sprints in the field compared to the laboratory environment. The authors concluded that the large increases in force and peak power were due to actual cycling locomotion, which enabled natural medial and lateral oscillations of the bike in field-testing.

In contrast, Gardner et al. (10) analysed the relationship between laboratory and field results in seven international track sprint riders. The riders performed two maximal $6 \mathrm{~s}$ cycle ergometer sprints and two $65 \mathrm{~m}$ standing starts on a standard track bike. No statistical differences were reported between maximum torque, maximum power and optimal pedalling rate in the laboratory versus field-testing. These findings led the authors to conclude that as the laboratory data and field-testing data presented no statistical differences they could be used interchangeably.

More recently Karsten et al. (18) conducted a laboratory versus field study to ascertain the validity of field-testing power output. Karsten et al. (18) analysed 14 trained cyclists on a laboratory cycle ergometer and on an indoor 
Velodrome. This study reported a high level of agreement (LoA - 10.98-10.8 W) between the power outputs in the field $(234 \pm 24 \mathrm{~W})$ when compared to findings from the laboratory (234 $\pm 25 \mathrm{~W})$. These findings further support those of Gardener et al. (10) as the two environments did not show a statistically significant difference.

To the authors knowledge Bertucci and Hourde (4) have conducted the only BMX study utilising laboratory and field-testing environments. The research examined the percentage contribution of upper and lower body on power production. In the study Bertucci and Hourde (4) also identified a number of physical factors that can influence the outcome of BMX performance: peak power, time of power production and torque. However, the study did not investigate any possible variations between the performance factors obtained in the two environments. Consequently, any variation in the measurement of peak power, torque and time of power production amongst BMX riders in the laboratory versus field are unknown.

Therefore, due to the limited peer reviewed data the aim of the current study is to ascertain any variation in peak power, torque and time of power production between the two environments in BMX cyclists. It is anticipated the results will enable BMX coaches and researchers to make an informed decision about the validity of data collected in the laboratory and field environment.

\section{METHODS}


Seventeen riders in the United Kingdom currently hold an elite level licence and from this population eight elite male BMX riders volunteered to take part in the study (mean age $21 \pm 2 \mathrm{yrs}$ ). Stature of the riders was recorded using a Harpenden stadiometer (Cranlea, UK) to the nearest $0.1 \mathrm{~cm}$, whilst body mass and percentage body fat were recorded using air displacement plethysmography (Bod Pod, Life Systems International, USA). The riders mean characteristics were, stature $170 \pm 6 \mathrm{~cm}$, body mass $69 \pm 3 \mathrm{~kg}$ and body fat $10 \pm 2 \%$. All the riders had previous experience of using laboratory SRM cycle ergometers and had ridden the Manchester BMX track. Written consent was obtained from all participants and a detailed description of the test protocol was issued to all participants prior to the study. The research protocol and experimental design received ethical approval from the University of Derby Ethics Human Studies Board and in accordance with the Declaration of Helsinki.

\section{Experimental Design}

In order to establish the validity of analysing a BMX rider's peak power, time of power production and torque in a laboratory and in the field two separate trials were conducted.

The first trials were conducted in a laboratory environment. Each subject performed three repeated sprint tests on a Laboratory Schoberer Rad Messtechnik (SRM) using a 32 strange gauge cycle ergometer. The ergometer was adapted to more accurately mimic a BMX bike used in competition. This was achieved through attaching a standard $70 \mathrm{~cm}$ straight bar to the ergometer, along with Shimano SPD (Shimano 
Pedal Dynamics: Shimano INC, Osaka, Japan) pedals, and altering the crank arm length to $175 \mathrm{~cm}$. Prior to testing each rider adjusted the bar height and stem length to their preferred position. The saddle was lowered so it did not interfere with the riders when performing each sprint. An inertial load of $50.2 \mathrm{~kg} \cdot \mathrm{m}^{2}$ was added to the cycle ergometer which according to Debraux et al. (6) equates to a standard BMX gear ratio of 43 (front chain ring)/16 (rear chain ring). Prior to the testing protocol each rider performed their standard pre-competition warm-up consisting of seated cycling, and a series of standing short sprints. Each rider then performed three 10second sprints in a standing position and was encouraged to reach maximal power in the shortest possible time duration. The riders were instructed to perform the sprints from a stationary position with their self-selected leg in the lead position. A rest period of ten minutes (23) was given between tests. Data from all eight riders' sprints were recorded using SRMWin software version 7.

The second trials were undertaken at the British National Indoors BMX Centre, Manchester, three weeks after the initial laboratory trials. The indoor track has a 5 meter high start ramp with a $28^{\circ}$ decent. The track measures 400 meters in length, has 4 straights with a number of technical jumps on each straight section, and three berms (corners).

The riders performed a structured self-paced warm-up consisting of a series of standing short sprints. Riders' then performed three 10-second sprint tests from the 5 meter high start ramp using a standard electronic start gate (Pro-Gate, Rockford, Illinoi, USA). The riders used their own cycles, which were all fitted with an (SRM) 8 
strange gauge crank and a standard gear ratio of $43 / 16$. Data from all eight riders' sprints were recorded using SRMWin software version 7.

Data analysis

Power and cadence data from both the laboratory and SRM crank power meter was used to calculate the rider's peak torque. Torque $(\mathrm{T})$ was calculated as: $T=P /(R \times \pi / 30)$, where $P$ is power; $T$ is torque and $R$ is cadence.

Descriptive statistics were used to analyse the percentage difference between the laboratory and field tests. A paired samples $t$ - test was used to calculate variations between power, torque, time to peak power in the laboratory and field and the alpha value was set at $p=<0.05$.

Agreement was established using $95 \%$ limits of agreement $(5,1)$. Differences between the two measures were plotted against the mean values and analysed for heteroscedasticity (see Figures 1, 2, 3). Where this was evident, data were logarithmically transformed to calculate the ratio limits of agreement.

\section{RESULTS}

The main purpose of this study was to examine the validity of testing elite BMX rider's peak power, torque and time of power production in a laboratory and comparing this data with testing conducted in a field-based environment. The results below provide a detailed breakdown for each of the individual BMX riders. Using a paired samples $t$ - test the results revealed significant differences between the laboratory testing and field testing of peak power $t(7)=-11.38(p=<0.01)$ and the 
relationship between the two environments was estimated as $r=0.78$. The relationship between the laboratory and field-testing time of power production was estimated as $r=0.86$ and also reported significant differences, $t(7)=8.64(p=<$ 0.01). However, no significant difference existed between torque in the laboratory verses field test $t(7)=-1.48(p=0.18) r=0.61$.

|//////////////////////////////// INSERT TABLE 1/////////////////////////////////////////////////////

Figure 1 demonstrates the limits of agreement for peak power between the laboratory and field-testing. The upper limits of agreement estimate at between 246 $714 \mathrm{~W}$ showing that a greater peak power is produced in field testing than the laboratory.

|||||||||||||||||||||||||||||||||||||| INSERT FIGURE 1 |||||||||||||||||||||||||||||||||||||||||||||||||||||||||||||||

Figure 2 below shows the limits of agreement for time to peak power between the laboratory and the field-testing. The riders were able to reduce the time of peak power production in the field compared to the laboratory with lower limits of agreement of -3.39 to $-0.73 \mathrm{~s}$.

||||||||||||||||||||||||||||||||||||||| INSERT FIGURE 2 |||||||||||||||||||||||||||||||||||||||||||||||||||||||||||||||

Figure 3 demonstrates the limits of agreement for peak torque between the laboratory and field-testing environments. The limits of agreement for torque were - 
32.99 to 19.07 for the lower and upper limits respectively. These limits reveal slightly higher torque values in the laboratory, but they were not statistically significant. $(p=$ $0.18)$.

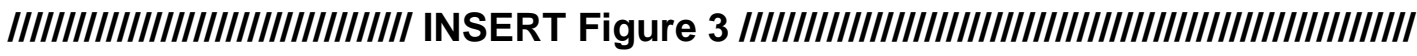

\section{DISCUSSION}

The purpose of the current study was to determine any variations in data collected in a laboratory compared to that in a field-testing environment amongst elite BMX riders. The results revealed that peak power was significantly higher $(p=<0.01)$ in the field tests when compared to the data recorded in the laboratory, with values of $34 \pm 9 \%$. Additionally, time of power production was significantly reduced $(p=<$ 0.01 ) in the field environment by $105 \pm 24 \%$. These results therefore pose the following questions (a) Is the field data over-estimating the values recorded? (b) Is the laboratory under estimating the values recorded?

In an attempt to answer these questions it is worth considering the findings of Herman et al. (14) who analysed time of peak power production and peak power in five elite BMX riders including two Olympic medallists. They analysed the standing start sprint results from the five riders performed on a flat paved surface. The results showed a high peak power of $2087 \pm 157 \mathrm{~W}$ and a reduction in time of power production - $1.6 \mathrm{~s}$, which have a closer comparison to the field data in this study than the laboratory data. In a separate study Debraux et al. (6) also found high power outputs in seven elite BMX riders who performed standing starts on a flat asphalt surface. The riders in his study were recorded to have similar peak powers $(1631 \pm$ 
$368 \mathrm{~W})$ as the field results from the current study $(1671 \pm 188 \mathrm{~W})$. Although Herman et al. (15) and Debraux et al. (6) did not undertake their research on a BMX track, both studies established that peak power values were higher (2087 $\pm 156.8 \mathrm{~W}, 1631$ $\pm 368 \mathrm{~W}$ respectively) than the laboratory data recorded in the current study (1191 \pm $188 \mathrm{~W})$.

One possible explanation for the high field values may be the contribution of upper body to force development in BMX cyclists. Several studies have examined the effect of upper body muscle activation on power and performance in cycling $(7,21,16,12)$. They all concluded that upper body activation had a statistically significant influence on performance. To enable riders in this study to use their upper body effectively in the laboratory testing, the SRM cycle ergometer was adjusted to accurately replicate the field riding position. The standard SRM cycle ergometer road bars were removed and replaced with a $70 \mathrm{~cm}$ flat bar. Each rider then adjusted the stem length and height of the ergometer to their preferred position. However, the riders produced $480 \pm 119$ less Watts on the ergometer compared to riding their own bikes on the BMX track. A possible explanation for this reduction in peak power was presented in a study by Bertucci et al. (6). The authors inferred that BMX riders rely on the upper body to produce power disproportionally compared to other cycling disciplines.

BMX riders use their upper body by oscillation of the bike and associative leverage. As the ergometer is a static rigid piece of equipment riders were unable to oscillate during sprints. This could be a major factor in explaining the difference in power and torque. However, this does not explain the reduction in time of power production. A 
possible explanation for this decrease in time of power production may be due to the geometry of the track. The gravitational force acting on the rider may be increased as the riders descend the $28^{\circ}$ start ramp. However, this was unfortunately outside the remit of this particular study.

\section{PRACTICAL APPLICATION}

The data in the current study suggests significantly lower results are recorded in the laboratory testing environment, when compared to the field testing environment. These findings have implications when comparing power and time of peak power production research data conducted in a laboratory to field data, as well as the utilisation of laboratory data for competitive benchmarking. Crucially, this does not mean that data collected in a laboratory cannot be used. Instead the application of BMX riders' data used interchangeable between the laboratory and the field should be viewed with an amount of caution. Based on the discussion in this paper it may be useful to investigate the contribution of a BMX riders' upper body on performance variables such as velocity in a race.

\section{ACKNOWLEDGEMENTS}

The authors gratefully acknowledge the British Cycling Federation Coach Jeremy Hayes for his time and dedication to the research project. There are no conflicts of interest for this work.

\section{REFERENCES}

1. Atkinson, G, and Nevill, AM. Statistical methods for assessing measurement error (reliability) in variables relevant to sports medicine. Sports medicine 26: 217-238, 1998. 
2. Balmer, JAMES, Davison, RR, and Bird, SR. Peak power predicts performance power during an outdoor 16.1-km cycling time trial. Medicine and Science in Sports and Exercise 32: 1485-1490, 2000.

3. Bertucci, W, Taiar, R, and Grappe, F. Differences between sprint tests under laboratory and actual cycling conditions. Journal of Sports Science and Medicine 45: 277-83, 2005.

4. Bertucci, $W$, and Hourde, C. Laboratory testing and field performance in BMX riders. Journal of Sports Science and Medicine 10: 417-419, 2011

5. Bland, J M, and Altman, DG. Statistical methods for assessing agreement between two methods of clinical measurement. Lancet 1, 307-210, 1986.

6. Debraux, P, Manolova, AV, Soudain-Pineau, M, Hourde, C, and Bertucci, W. Maximal torque and power pedaling rate relationships for high level BMX riders in field tests. Journal of Science in Cycling 2: 51-57, 2013.

7. Doré, E, Baker, SJ, Jammes, A, Graham, M, New, K, and Van Praagh, E. Upper body contribution during leg cycling peak power in teenage boys and girls. Research in Sports Medicine 14: 245-257, 2006.

8. Eriksson, JS, Rosdahl, H, and Schantz, P. Validity of the Oxycon Mobile metabolic system under field measuring conditions. European Journal of Applied Physiology 112: 345-355, 2012.

9. Faria, EW, Parker, DL, and Faria, IE. The science of cycling: Physiology and training - Part 1. Sports Medicine 35: 285 - 312, 2005.

10. Gardner, SA, Martin, JC, Martin, DT, Barras, M, and Jenkins, DG. Maximal torque- and power-pedaling rate relationships for elite sprint cyclists in laboratory and field tests. European Journal of Applied Physiology 101: 287292, 2007.

11. Goosey-Tolfrey, VL, and Leicht, CA. Field-based physiological testing of wheelchair athletes. Sports medicine 4: 77-91, 2013. 
12. Grant, MC, Roberts, R, Baird, MF, and Baker, JS. The Effect of Prior Upper Body Exercise on Subsequent Wingate Performance. BioMed Research International, 2014.

13. Hawley, JA, \& Noakes, TD. Peak power output predicts maximal oxygen uptake and performance time in trained cyclists. European Journal of Applied Physiology and Occupational Physiology 65: 79-83, 1992.

14. Herman, CW, McGregor, SJ, Allen, H, and Bolt, EM. Power Capabilities of Elite Bicycle Motocross Racers During Field Testing In Preparation For 2008 Olympics. Medicine \& Science in Sports \& Exercise 41: 306-307, 2009.

15. Hopker, J, and Jobson, S. (Eds.) Performance Cycling: The Science of Success. A\&C Black, 2012.

16. Hurst, HT, Swarén, M, Hébert-Losier, K, Ericsson, F, Sinclair, J, Atkins, S, and Holmberg, HC. Influence of course type on upper body muscle activity in elite cross-country and downhill mountain bikers during off road downhill cycling. Journal of Science and Cycling 1: 2-9, 2012.

17. Jobson, SA, Nevill, AM, Palmer, GS, and Jeukendrup, AE. The ecological validity of laboratory cycling: Does body size explain the difference between laboratory- and field-based cycling performance? Journal of Sports Sciences 25: $3-9,2007$.

18. Karsten, B, Jobson, SA, Hopker, J, Jimenez, A, and Beedie, C. High agreement between laboratory and field estimates of critical power in cycling. International Journal of Sports Medicine 35: 298-303, 2014.

19. Padilla, S, Mujika, I, Angulo, F, and Goiriena, JJ. Scientific approach to the 1h cycling world record: a case study. Journal of Applied Physiology 89: $1522-$ 1527, 2000.

20. Palmer, GS, Dennis, SC, Noakes, TD, and Hawley, JA. Assessment of the reproducibility of performance testing on an air-braked cycle ergometer. International Journal of Sports Medicine 17: 293 - 298, 1996. 
21. Segerström, ÅB, Holmbäck, AM, Elzyri, T, Eriksson, KF, Ringsberg, K, Groop L, Ola Thorsson, O, and Wollmer. P. Upper body muscle strength and endurance in relation to peak exercise capacity during cycling in healthy sedentary male subjects. The Journal of Strength \& Conditioning Research 25: 1413-1417. 2011.

22. Smith, MF, Davison, RCR., Balme, RJ, and Bird, S.R. Reliability of mean power recorded during indoor and outdoor self-paced $40 \mathrm{~km}$ cycling timetrials. International Journal of Sports Medicine 22: 270 - 274, 2001.

23. Thatcher, R, Gifford, R, and Howatson, G. The Influence of Recovery Duration After Heavy Resistance Exercise on Sprint Cycling Performance. The Journal of Strength \& Conditioning Research 26: 3089-3094, 2012.

24. Waldron, M, Worsfold, P, Twist, C, and Lamb, K. Concurrent validity and testretest reliability of a global positioning system (GPS) and timing gates to assess sprint performance variables. Journal of Sports Sciences 29: 16131619, 2011.

25. Wooles, AL, Robinson, AJ, and Keen, PS. A static method for obtaining a calibration factor for SRM bicycle power cranks. Sports Engineering 8: 137144, 2005. 
Table 1

\begin{tabular}{lccc}
\hline & $\begin{array}{c}\text { Peak power } \\
(\mathrm{W})\end{array}$ & $\begin{array}{c}\text { Time to Peak } \\
\text { Power }(\mathrm{s})\end{array}$ & Peak Torque $(\mathrm{Nm})$ \\
\hline Field & $1671 \pm 188$ & $1 \pm 1$ & $117 \pm 15$ \\
Laboratory & $1191 \pm 188$ & $3 \pm 1$ & $124 \pm 15$ \\
Difference & $480 \pm 119$ & $-2 \pm 1$ & $-7 \pm 13$ \\
Difference (\%) & $34 \pm 9$ & $105 \pm 24$ & $6 \pm 8$ \\
\hline
\end{tabular}

Table 1 shows the combined results for peak power, time of power production and peak torque for all eight riders in both the laboratory and field tests.

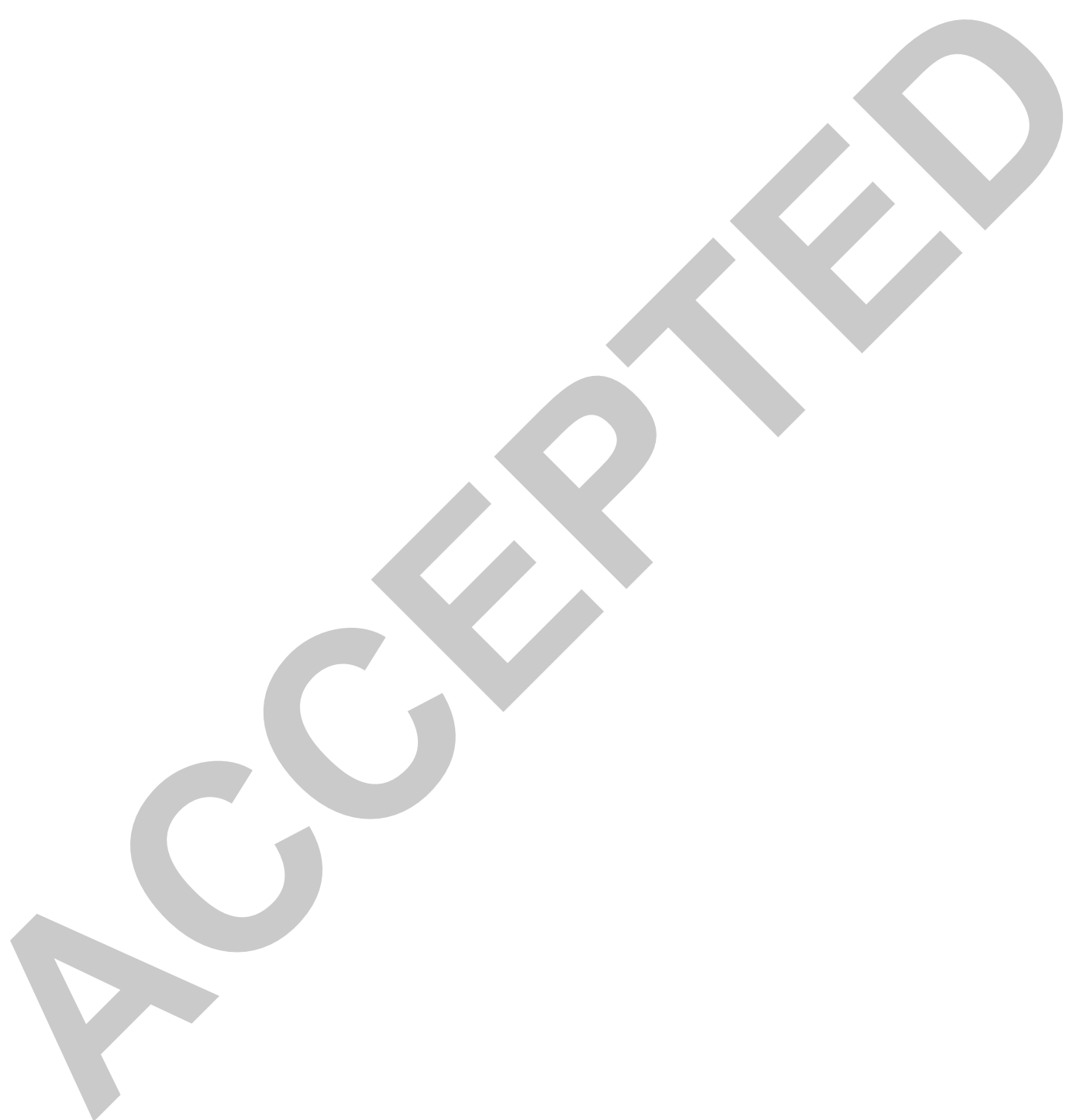


Figure 1

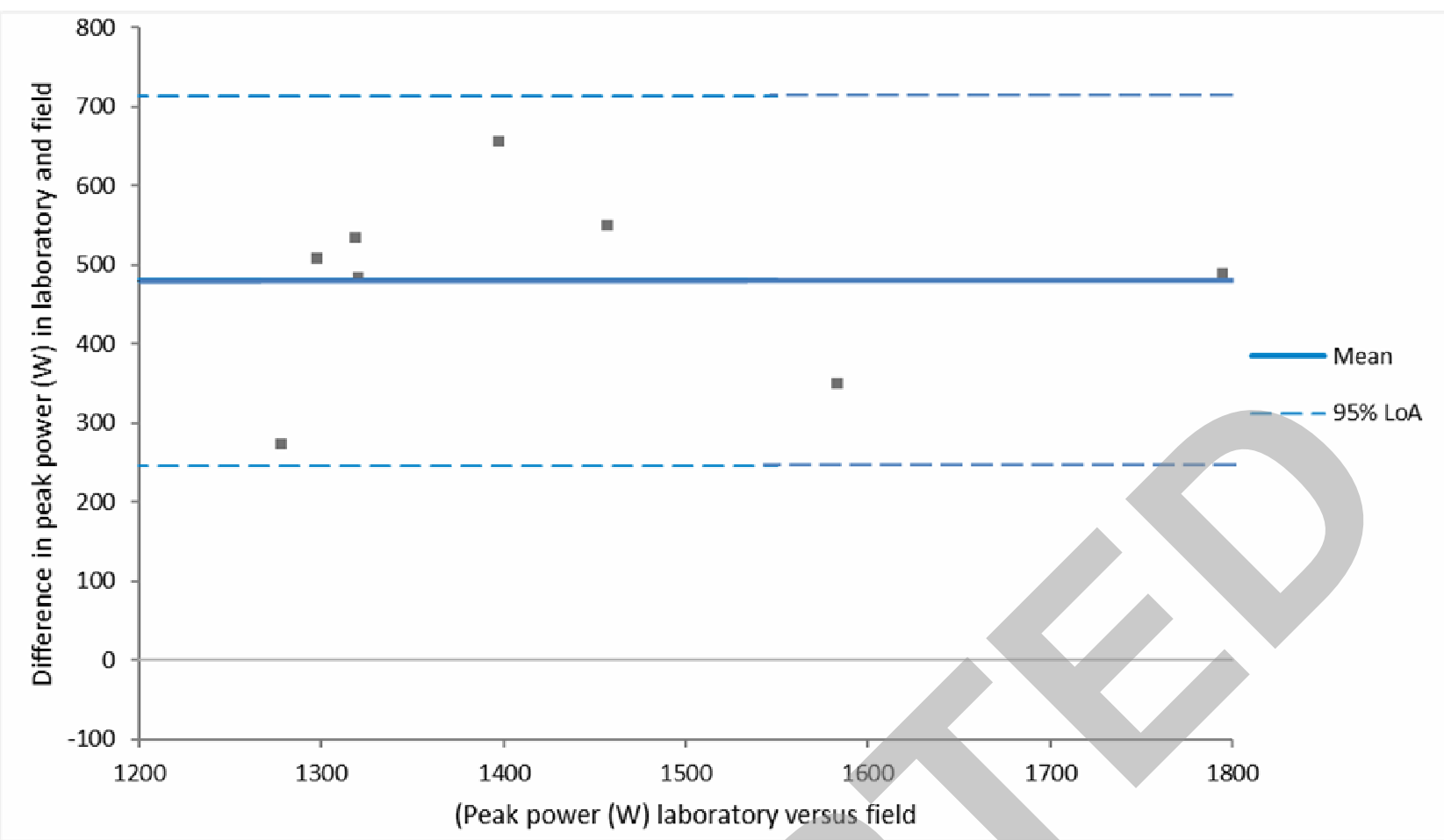

Figure 1- Bland and Altman plot for limits of agreement peak power (W) between laboratory and field testing $\left(Y=0.7964 x-139.95 R^{2}=0.6382\right)$

\section{Figure 2}

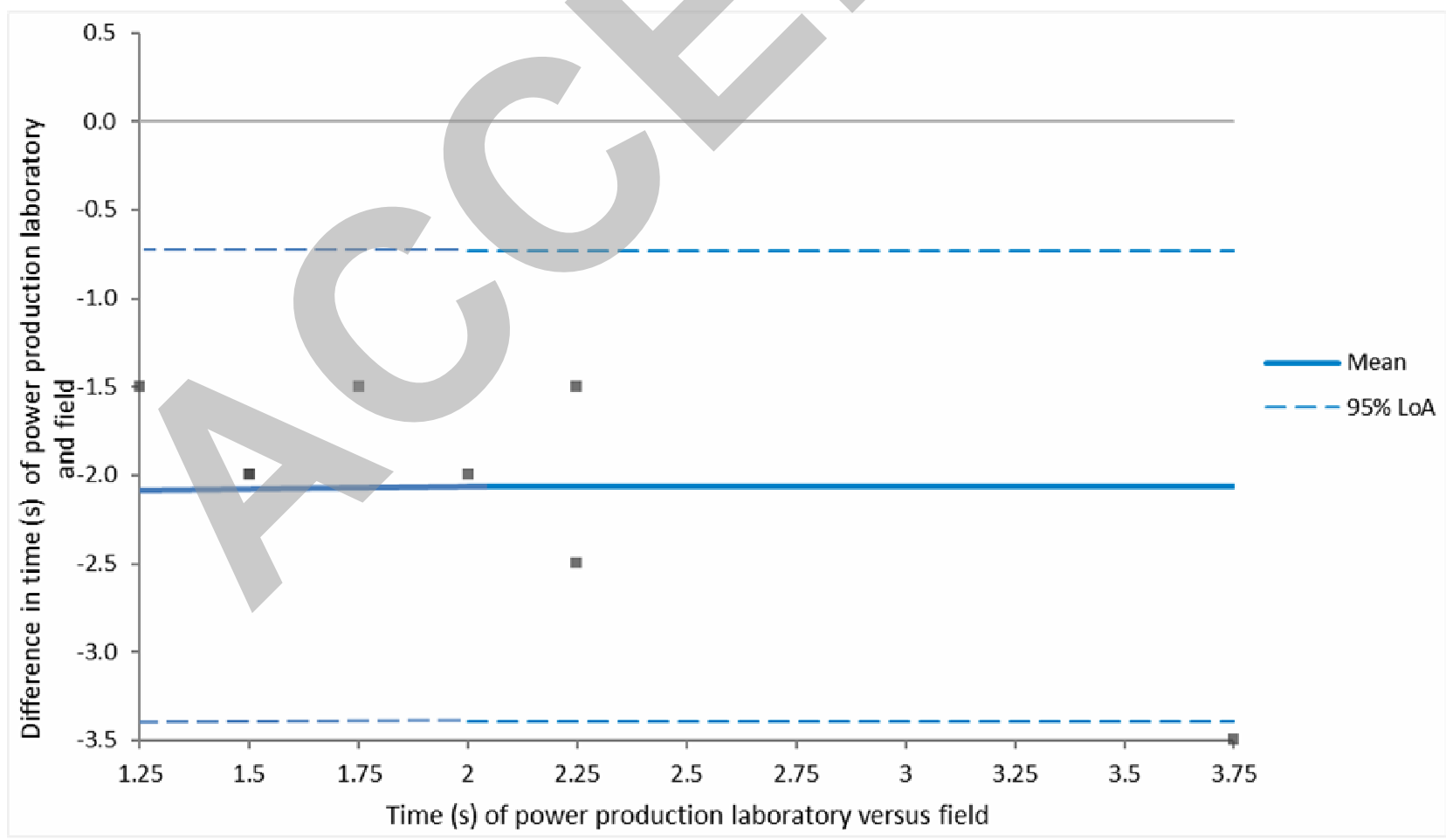

Figure 2-Bland and Altman plot for limits of agreement in time (s) of power production (W) between laboratory and field testing $\left(y=0.42959 x-0.3042 R^{2}=0.7452\right)$ 


\section{Figure 3}

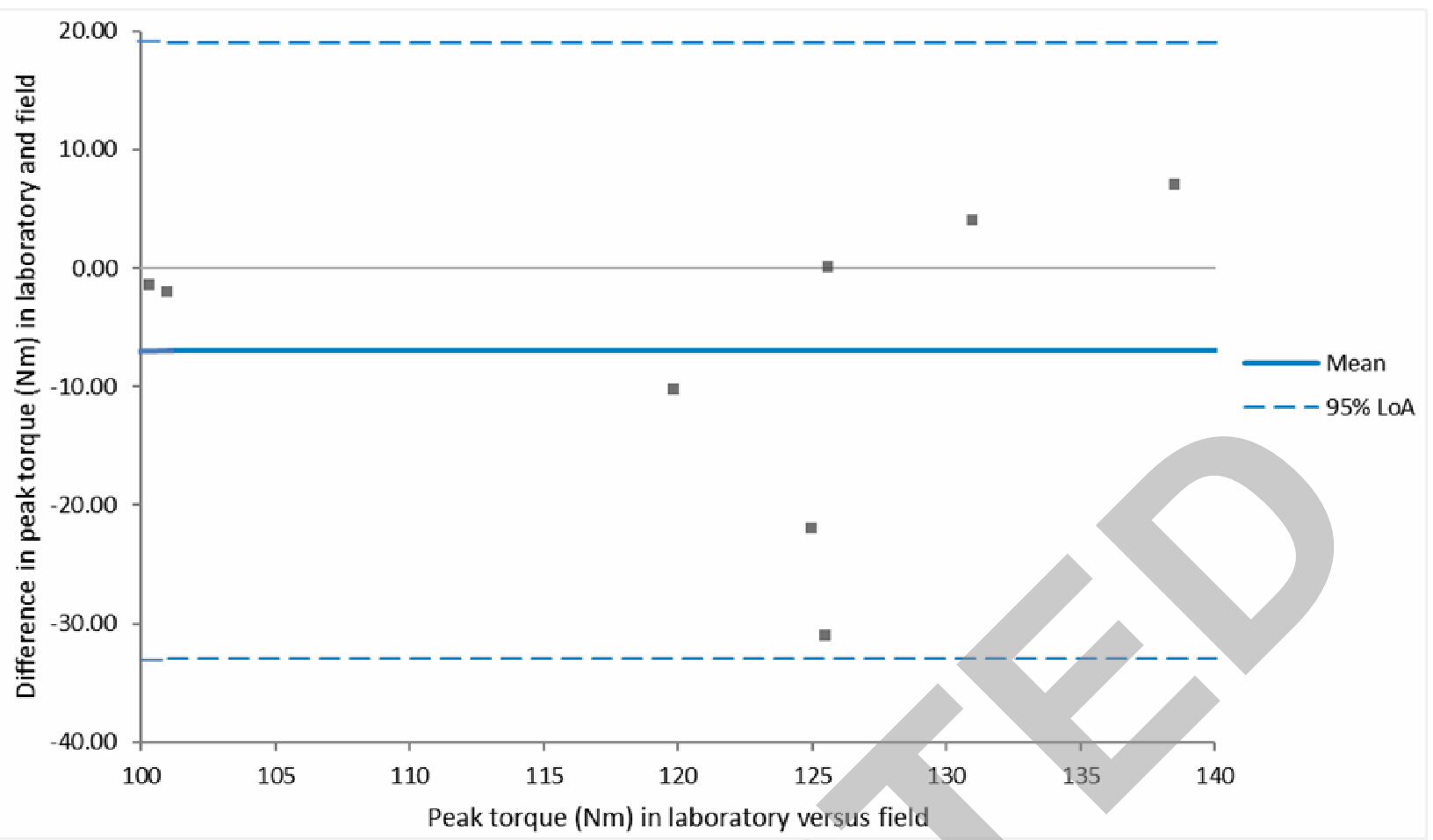

Figure 3-Bland and Altman plot for limits of agreement in peak torque $(\mathrm{Nm})$ between laboratory and field testing $\left(y=0.6175 x+40.594 R^{2}=0.3779\right)$ 